\title{
Health related quality of life in individuals with cognitive decline and discrepancies between patients and their proxies
}

\author{
Iban Onandia-Hinchado $^{\mathrm{a}, *}$, Unai Diaz-Orueta ${ }^{\mathrm{b}}$

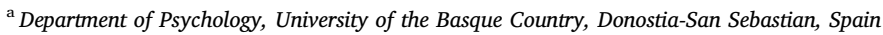 \\ ${ }^{\mathrm{b}}$ Department of Psychology, Maynooth University, Maynooth, Co. Kildare, Ireland
}

\section{A R T I C L E I N F O}

\section{Keywords:}

Dementia

HRQoL

Patient rated outcomes

Caregiver rated outcomes

Quality of life

SF-12

\begin{abstract}
A B S T R A C T
Background and Objective: The goal was to measure Health Related Quality of Life (HRQoL) in individuals with different types of dementia, mild cognitive impairment (MCI) and healthy controls (HC), and assess agreement levels between participants and proxies.

Materials and Methods: A sample of 136 participants were recruited; thirty-seven with Alzheimer's Disease Dementia (AD), 19 with Dementia with Lewy Bodies (DLB), 12 with Frontotemporal Dementia (FTD), 37 with MCI and 31 HC. HRQoL was measured via the 12-Item Short Form Health Survey, version 2 (SF-12-v2), separately for participants and proxies. Two groups (Individuals with cognitive decline versus healthy controls) were matched for sociodemographic variables. Differences for discrepancy rates between both groups were measured using t-test. Participant and proxy agreements were measured via Intraclass Correlation Coefficients (ICC). Linear regression analyses were performed to examine what variables explained better the variance observed. Results: Patients with DLB and FTD showed the lowest levels of HRQoL, while AD and HC showed the highest. No statistically significant differences were found between discrepancy scores from cognitive decline groups and healthy controls. ICC indicated high agreement between patients and proxies for the groups with cognitive decline, while agreements achieved in HC were lower and only in physical indices. GDS score accounted for $8.3 \%$ of the variance of proxies' rating on Mental Summary Composite Score (MSC).

Conclusion: HRQoL physical and mental summaries are more reliable in groups with cognitive decline. Healthy controls and their proxies show lower agreement, with proxies reporting lower levels than normal for some relevant indices.
\end{abstract}

\section{Introduction}

In the latest 10-15 years, dementia has been a target condition for studies that have developed treatments to improve cognitive status, delay severe symptoms onset, maximize daily life functioning of patients and reduce behavioural disorders. However, despite the wide variety of approaches for dementia patients, the outcomes only show moderate rates of success (Logsdon, Gibbins, McCurry, \& Teri, 2002). Moreover, new challenges and targets have raised not only in relation to the patients with dementia themselves, but also to their environment (Gwyther, 1997). Variables such as Health Related Quality of Life (HRQoL) are essential for a comprehensive approach of the disease, and very useful to inform about disease progression, such as cost-effectiveness models on dementia. Indeed, it is recognized as a high valued health outcome measure in dementias (Landeiro, Walsh, \& Ghinai, 2018).
World Health Organization defines HRQoL as the personal perception with regards to one's own vital position in relation to his/her achievements, expectations, standards and concerns (Sartorius, 1990s). There are three levels of HRQoL measurement: (1) by means of disorder specific instruments, (2) finding generic health profiles, and (3) by means of studies using a cost-utility analysis (Wlodarzcyk, Brodaty, \& Hawthorne, 2004). Up to date, most studies on this area have focused their efforts in the conceptual analysis of HRQoL in dementia, as well as in the study of psychometric properties of selected developed instruments, thus showing evidence of the relationship between HRQoL and clinical variables such as cognition, limitations in Daily Life Activities, behavioural disorders, depression or caregiver burden (Banerjee, Samsi, \& Petrie, 2009). Subsequently, and due to the wide extension of symptoms associated to dementia, there is an increasing consensus on the need to measure variables and constructs such as HRQoL, that need to be measured and given similar relevance to those related to cognition

\footnotetext{
* Corresponding author at: UPV/EHU, Facultad de Psicología, Avda. de Tolosa, 70, 20018 Donostia-San Sebastián, Spain.

E-mail address: iban.onandia@ehu.eus (I. Onandia-Hinchado).
} 
and behaviour (Rabins \& Black, 2007; Whitehouse, 2000). The implicit difficulty to measure this variable lies on its dependency on the patient's communication skills to express their impressions to the evaluator, as well as on the tendency to be compliant with the evaluator (Wlodarzcyk et al., 2004). Measurements of HRQoL are useful not only when it comes to analyse the impact of the disease on patients, but also when there is a need to objectivize the effects of intervention, according to Schwartz (2013). This same author emphasizes the usefulness of this type of measurements, not only for the design of services and resources for people with cognitive decline who enter a nursing home, but also for the establishment of priorities and treatment requirements.

The Mini Mental State Examination (MMSE) score does not correlate with HRQoL self-perception, or with caregivers' reports (Logsdon et al., 2002; Vogel, Mortensen, Hasselbalch, Andersen, \& Waldemar, 2006). Age and behavioural disorders, on the contrary, substantially influence HRQoL rates reported by caregivers in dementia (Banerjee, Smith, \& Lamping, 2006, 2009), while these variables do not seem to affect patients' ratings. This raises the need to evaluate the differential impact that dementia may have on patients and caregivers, and to consider how the decline of awareness in dementia may affect the outcomes (Banerjee et al., 2006; Logsdon et al., 2002). Other variables affecting HRQoL are: (1) gender (Woods, Thorgrimsen, Spector, Royan, \& Orrell, 2006); (2) depressive disorders (Logsdon et al., 2002), (3) anxiety disorders (Banerjee et al., 2006), whose treatment considerable improves HRQoL rates (Woods et al., 2006) and (4) functional status (Karlawish, Lu, Logsdon, Whitehouse, \& Aisen, 2004).

In a longitudinal study with 47 patients with dementia, only a lower baseline score in an Alzheimer specific quality of life scale was associated with greater decline in the follow-up, with no associations between sociodemographic variables, baseline ratings of dementia severity (MMSE), impairment in activities of Daily Living (ADL), behavioural impairment, depression, or MMSE change during follow-up (Lyketsos et al., 2003). When early interventions take place, low baseline scores can improve dramatically (Banerjee et al., 2007; Zimmerman, Sloane, \& Williams, 2005). By implementing a cognitive stimulation therapy on a group basis, studies report improvements when compared to control groups (Spector, Thorgrimsen, \& Woods, 2003), though their maintenance does not last for long and HRQoL scores decrease together with MMSE scores (Orrell, Spector, Thorgrimsen, \& Woods, 2005). Female patients seem to benefit more from intervention, while male patients lacking any treatment perform worse (Woods et al., 2006).

When comparing quality of life among chronic diseases, HRQoL in individuals with dementia could appear relatively preserved. Nevertheless, when sociodemographic variables are taken into account, patients with dementia experience the largest decline. Moreover, quality of life declines with advanced age, while dementias develop more frequently in late ages (Esteban y Peña, Hernández, \& Fernández, 2010). The type of dementia also affects HRQoL scores, with worse ratings for Dementia with Lewy Bodies (DLB) (Banerjee et al., 2009).

The main methodological challenge relies on the subjectiveness of HRQoL measures, especially when self-reporting may be especially compromised in conditions affecting insight abilities, emotional and affective skills, and in which a tendency to distort reality may take place (Wlodarzcyk et al., 2004). Many studies have pointed out the need to collect informants' views, although other studies state that these views may be biased and that patients' own views and reports should be prioritized (Herrman, Hawthorne, \& Thomas, 2002).

While some studies suggest that HRQoL measurements are only possible with mild to moderate dementia, it seems that proxies, both formal and informal caregivers, share a different opinion. Consistent associations were found between proxies' report and cognitive decline, decrease in activity participation, depression and agitation (Sloane et al., 2005), while no associations were found based on patients' selfreports. Some features of the proxies (overburden, depression, educational level and support from professional caregivers) influence their view on patients' HRQoL, being their ratings significantly worse than those of patients' themselves (Vogel et al., 2006). Although frequently it is stated that this may be due to dementia patients' low comprehension skills and caregivers' high level of criticism, Evans and Huxley (2005) state that this is because dementia implies a process of adaptation to a disability in which people with dementia mark and adjust expectations and responses to changes. In fact, this discrepancy was not only reported for dementia.

Albrecht and Devilieger (Albrecht \& Devlieger, 1999) already coined the term 'disability paradox' to refer to the fact that half of the people with disabilities report a good or even excellent quality of life. In the case of caregivers of individuals with dementia, chronicity and long development of the disease, linked to a bad prognosis, prevent this paradox to take place and, instead, increase significantly caregivers' stress levels (Clipp \& George, 1993). A way to minimize the impact of their own stress when responding to HRQoL questionnaires is to instruct the proxy to answer as if they were the person with dementia (Karlawish, Casarett, Klocinski, \& Clark, 2001). Both perspectives seem to conform complementary approaches to HRQoL (Banerjee et al., 2009).

Latest studies show that data for dementia specific HRQoL measures can be obtained using subjective reports from patients and proxies (Logsdon et al., 2002). Measures are quick and easy to use, and have been tested in a variety of cases and situations (Banerjee et al., 2009). The National Institute for Health and Clinical Excellence states and proves an improvement of HRQoL measures and their usefulness for intervention in dementia cases (National Institute for Health \& Care Excellence, 2006; Winblad, Brodaty, \& Gauthier, 2001). In this sense, it is of great relevance to redefine what constitutes treatment success in order to incorporate patient's lifestyle variables.

With this idea of the relevance of HRQoL for dementia treatment planning, the goal of the current study was to measure self-reported Health Related Quality of Life (HRQoL) in individuals with different types of dementia, mild cognitive impairment (MCI) and healthy controls (HC), as well as those reports delivered by their proxies (relatives, caregivers...), and to assess agreement levels between participants and their proxies. We hypothesized, based on previous research, the existence of high levels of discrepancy between participants and proxies across all HRQoL domains.

\section{Method}

The study comprised an incidental sample of 136 participants divided in 5 groups: (a) 37 individuals with a diagnosis of Alzheimer's Dementia (AD); (b) 19 individuals with a diagnosis of Dementia with Lewy Bodies -DLB-; (c) 12 individuals with Frontotemporal Dementia-FTD- and (d) 37 individuals with a diagnosis of Mild Cognitive Impairment -MCI-. Moreover, a control group was available: (e) 31 individuals with no cognitive decline, namely Healthy Controls -HC-, recruited from older adults' associations in the same geographical area, and that were matched with the rest of the groups based on age, gender, years of education and family history of primary degenerative dementias (thus, HC did not differ significantly from clinical groups in any of the grouping variables). People who attended a neurology clinic and fulfilled the inclusion criteria were invited to voluntarily participate in NeuroDemeNPSia study, in which cross-sectional measures of psychological and neuropsychological variables were taken. Inclusion criteria included:

(1) cognitive decline due to a degenerative primary dementia (AD, DLB or FTD) (with scores in the GDS scale of 3 or 4) (Reisberg, Ferris, de Leon, \& Crook, 1982), or due to a mild cognitive impairment but not dementia (MCI). Those scoring 1 or 2 in the Global Deterioration Scale (GDS) and showing a regular cognitive performance (meaning that they are not above -i.e. super agers that may constitute as outliers - or below two standard deviations from the mean 
-i.e. individuals with MCI or dementia - in the administered cognitive tests) were included as HC;

(2) individuals with a diagnosis of AD had to fulfil National Institute of Neurological and Communicative Disorders and Stroke and the Alzheimer's Disease and Related Disorders Association (NINCDSADRDA) diagnostic criteria (Dubois, Feldman, \& Jacova, 2007); those with DLB had to fulfil DLB International Workshop diagnostic criteria (McKeith, Dickson, \& Lowe, 2005); those with FTD had to fulfil the criteria from the Work Group Frontotemporal Dementia and Pick's Disease (McKhann et al., 2001). Individuals with MCI had a score of 3 in the GDS and fulfil criteria from the Spanish Neurological Society (SEN) (Robles, Del Ser, Alom, Peña Casanova, \& grupo asesor del GNCD de la SEN, 2002), which imply (a) an impairment in one or more cognitive areas -attention/concentration, language, gnosias, memory, praxias, visuospatial and executive functions-; (b) this impairment has to be acquired, reported by the patient or a reliable proxy, objectivized via neuropsychological assessment, lasting for several months, and evidenced in the patient with a normal level of consciousness; (c) the cognitive impairment only interferes minimally with instrumental or advanced activities of daily living, and (d) the cognitive impairment is not associated with disorders on the level of consciousness;

(3) all received a diagnosis by a neurologist from a primary care setting in the Greater Bilbao area (Spain);

(4) all signed a consent form, with explicit authorization to participate given by the individual and, where required, from their caregiver.

Exclusion criteria followed in this study included: (1) suffering from conditions associated to decline in intellectual functions, such as cerebral infections, stroke, encephalic degenerative diseases, psychiatric disorders, or showing conditions affecting functionality and prevent their participation in the study (deafness, blindness...), all of it confirmed by the clinical record and a check-up by a neurologist; more specifically, those patients with any type of vascular factor were discarded in order to properly adjust to Primary Degenerative Dementia aetiology. It was specifically confirmed, patient by patient, by means of an fMRI no older than 6 months, that no individual had any issue suggesting vascular aetiology, but instead that fMRI results matched 'expected' results -i.e. fMRI within expected parameters of absence of vascular components; (2) those with problems to read or understand the contents of the administered questionnaires, and (3) those lacking a companion or informal caregiver that may respond as the main caregiver were also excluded. With regards to this, the main inclusion criteria for proxies was that they had known or met the participant frequently (on a daily or close to daily basis) for at least 6 months prior to the assessment. The study fulfilled all the ethical requirements and obtained the required ethical approval from the University of [blinded for review]

HRQoL was measured by means of the SF-12(v.2) questionnaire, developed by Ware, Kosinski, Turner-Bowker and Gandek (2005) and that includes 8 factors or subscales (Physical Function, Physical Role, Body Pain, General Health, Vitality, Social Function, Emotional Role and Mental Health) and 2 summary composite measures (physical-PSCand mental -MSC-). Scoring ranges from 0 to 100, where higher scores mean a better health-related quality of life. Research on this scale has shown internal consistency scores higher than 0.70 and significant correlations between the different versions of the scale (Jenkinson \& Layte, 1997), and a recent study with AD patients has shown alpha levels for different subscales ranging from 0.69 to 0.88 (Cole et al., 2014). The questionnaires were self-reported but the evaluator was present to clarify any doubts they might have, and if this was not enough, as reported above, those with reading or comprehension problems were excluded.

Norms and weights from the American version were used in absence of a full validation of the scale with Spanish population. Estimation of dimensions and summary composite scores was performed by means of the standard method that uses an algorithm with the mean scores, standard deviations and factorial weights in general population (Ware et al., 2005), leading to comparable typified scores. Thus, the algorithm generates, for each domain, a new variable with a mean of 50 (Standard Deviation of 10), which corresponds with the American norms mean through a linear transformation of punctuations, as other authors do with this instrument (Monteagudo, Hernando, \& Palomar, 2011). This way, the direct interpretation of results is easier (i.e. the higher the score, the better the HRQoL).

Data were analyzed using the Statistical Package for Social Sciences (SPSS), version 23.0 for Windows. The p-value was set in .05 . We compared HRQoL self-reports of patients with dementia with reports given by their main caregivers (proxies). To do that, we performed IntraClass Correlation (ICC) analyses, as well as $t$-test analyses to compare the clinical groups and control group. As defined by Shrout and Fleiss (1979), the ICC is the correlation between one measurement (either a single rating or a mean of several ratings) on a target and another measurement obtained on that target. In our case, a correlation between participants' and proxies' report was calculated. Moreover, linear regression analyses were performed in order to see what variables explained better the variance observed in the results (both for participants and proxies). More specifically, participants' age, GDS score and years of formal education were used as predictors, separately for proxies' and participants' ratings. Clinical groups were matched by sociodemographic variables, in order to control the effects of gender (one-tailed $p$-values $)\left(\chi^{2}=4.005 ; \mathrm{p}=.405\right), \quad$ age $\left(\chi^{2}=1.961\right.$; $\mathrm{p}=.161)$, years of formal education $\left(\chi^{2}=4.947 ; \mathrm{p}=.084\right)$ and family history of dementia (mother, $\chi^{2}=3.112 ; \mathrm{p}=.539$; father, $\chi^{2}=6.009$; $\mathrm{p}=.198$; siblings, $\chi^{2}=0.911 ; \mathrm{p}=.923$; paternal uncles, $\chi^{2}=3.881$; $\mathrm{p}=.422$; maternal uncles, $\chi^{2}=1.330 ; \mathrm{p}=.856$; paternal grandparents, $\chi^{2}=2.549 ; \mathrm{p}=.636$; maternal grandparents, $\chi^{2}=3.487$; $\mathrm{p}=.480$; partner, $\chi^{2}=5.367 ; \mathrm{p}=.252$; maternal cousin, $\chi^{2}=2.896$; $\mathrm{p}=.575$ and paternal cousin, $\chi^{2}=4.970 ; \mathrm{p}=.290$ ).

\section{Results}

Table 1 shows the sociodemographic variables and MMSE scores for all the participant subgroups. All groups had a mean age of around 75-76 years (with the exception of the MCI group, which, as would be expected, was younger). Individuals with $\mathrm{AD}$ showed fewer years of education than the rest of the groups. Regarding the general cognitive state measured by the MMSE, while the groups with dementia were below the cut point considered as cognitive impairment $(<24$ points, generally), the MCI group was in the range of suspicion for possible cognitive impairment (24-27 points) and healthy controls were above that range.

Table 1

Sociodemographic variables and MMSE scores.

\begin{tabular}{|c|c|c|c|c|c|c|}
\hline \multicolumn{2}{|c|}{ Variables } & \multirow{2}{*}{$\frac{\mathrm{AD}(\mathrm{n}=37)}{17(46 \%)}$} & \multirow{2}{*}{$\begin{array}{l}\text { LBD }(n=19) \\
10(52.63 \%)\end{array}$} & \multirow{2}{*}{$\begin{array}{l}\text { FTD }(n=12) \\
6(50 \%)\end{array}$} & \multirow{2}{*}{$\begin{array}{l}\text { MCI }(\mathrm{n}=37) \\
12(32.43 \%)\end{array}$} & \multirow{2}{*}{$\begin{array}{l}\text { HC }(\mathrm{n}=31) \\
9(29 \%)\end{array}$} \\
\hline Sex & Male N (\%) & & & & & \\
\hline & Female N (\%) & $20(54 \%)$ & $9(47.37 \%)$ & $6(50 \%)$ & $25(67.57 \%)$ & $22(71 \%)$ \\
\hline \multicolumn{2}{|c|}{ Age - $($ mean $\pm S D)$} & $76.66 \pm 6.59$ & $77.95 \pm 7.71$ & $75.77 \pm 6.21$ & $70.89 \pm 11.05$ & $74.68 \pm 6.35$ \\
\hline \multicolumn{2}{|c|}{ Years of education (mean $\pm S D$ ) } & $6.46 \pm 3.40$ & $7.44 \pm 2.68$ & $7.58 \pm 3.42$ & $9.64 \pm 5.53$ & $8.36 \pm 3.40$ \\
\hline \multicolumn{2}{|c|}{ MMSE score $($ mean $\pm S D)$} & $20.39 \pm 3.80$ & $21.63 \pm 3.99$ & $22.00 \pm 3.74$ & $25.41 \pm 3.18$ & $28.11 \pm 1.32$ \\
\hline
\end{tabular}


Table 2

HRQoL features of the sample.

\begin{tabular}{|c|c|c|c|c|c|}
\hline HRQoL Variables (Percentage \pm SD) & $\mathrm{AD}(\mathrm{n}=37)$ & DLB $(\mathrm{n}=19)$ & FTD $(n=12)$ & MCI (n = 37) & HC $(n=31)$ \\
\hline Physical Function (patient) & $83.97 \pm 30.61$ & $63.04 \pm 34.44$ & $69.64 \pm 41.81$ & $75 \pm 35.36$ & $80.65 \pm 27.16$ \\
\hline Physical Function (proxy) & $82.24 \pm 28.42$ & $52.38 \pm 32.50$ & $59.62 \pm 41.51$ & $68.92 \pm 36.52$ & $76.79 \pm 28.81$ \\
\hline Physical Role (patient) & $82.43 \pm 34.42$ & $52.98 \pm 43.28$ & $69.64 \pm 38.20$ & $69.23 \pm 36.93$ & $83.62 \pm 23.88$ \\
\hline Physical Role (proxy) & $61.84 \pm 28.47$ & $35.71 \pm 31.20$ & $33.65 \pm 32.43$ & $52.70 \pm 29.04$ & $63.39 \pm 21.50$ \\
\hline Body Pain (patient) & $90.38 \pm 24.07$ & $78.26 \pm 32.25$ & $82.14 \pm 31.67$ & $80.49 \pm 34.69$ & $80.65 \pm 33.98$ \\
\hline Body Pain (proxy) & $84.21 \pm 28.72$ & $77.38 \pm 35.27$ & $80.77 \pm 37.02$ & $75 \pm 32.81$ & $85.71 \pm 19.75$ \\
\hline General Health (patient) & $49.10 \pm 22.50$ & $37.17 \pm 27.71$ & $35.71 \pm 19.89$ & $50 \pm 25.12$ & $56.61 \pm 21.46$ \\
\hline General Health (proxy) & $51.71 \pm 23.49$ & $39.29 \pm 24.71$ & $35.77 \pm 32.14$ & $48.24 \pm 26.41$ & $62.32 \pm 25.15$ \\
\hline Vitality (patient) & $75 \pm 30.89$ & $64.77 \pm 37.53$ & $76.79 \pm 31.72$ & $54.89 \pm 38.41$ & $76.61 \pm 28.82$ \\
\hline Vitality (proxy) & $75 \pm 27.26$ & $73.81 \pm 26.78$ & $57.69 \pm 29.55$ & $74.32 \pm 27.94$ & $92.86 \pm 19.07$ \\
\hline Social Function (patient) & $93.57 \pm 19.50$ & $81.82 \pm 32.90$ & $87.50 \pm 23.51$ & $81.41 \pm 16.15$ & $90.18 \pm 26.65$ \\
\hline Social Function (proxy) & $65.13 \pm 22.16$ & $53.57 \pm 35.61$ & $55.77 \pm 32.52$ & $50.68 \pm 33.04$ & $65.18 \pm 21.88$ \\
\hline Emotional Role (patient) & $92.23 \pm 16.76$ & $65.48 \pm 41.44$ & $80.35 \pm 31.28$ & $81.09 \pm 31.13$ & $87.50 \pm 16.41$ \\
\hline Emotional Role (proxy) & $57.24 \pm 28.86$ & $47.62 \pm 30.78$ & $56.73 \pm 30.45$ & $55.07 \pm 28.63$ & $69.20 \pm 14.22$ \\
\hline Mental Health (patient) & $75.66 \pm 22.69$ & $68.18 \pm 24.92$ & $66.07 \pm 26.14$ & $69.82 \pm 28.23$ & $77.02 \pm 21.19$ \\
\hline Mental Health (proxy) & $66.12 \pm 16.92$ & $61.90 \pm 19.56$ & $60.58 \pm 18.29$ & $61.82 \pm 19.53$ & $76.34 \pm 13.75$ \\
\hline Physical summary (patient) & $49.13 \pm 11.57$ & $40.48 \pm 13.34$ & $44.23 \pm 13.14$ & $45.33 \pm 11.95$ & $48.10 \pm 11.23$ \\
\hline Physical summary (proxy) & $49.08 \pm 10.59$ & $41.57 \pm 9.20$ & $40.44 \pm 13.92$ & $45.08 \pm 10.09$ & $47.90 \pm 8.90$ \\
\hline Mental summary (patient) & $55.15 \pm 9.10$ & $50.65 \pm 11.41$ & $51.90 \pm 11.26$ & $50.12 \pm 12.36$ & $55.05 \pm 8.86$ \\
\hline Mental summary (proxy) & $44 \pm 9.98$ & $42.35 \pm 11.18$ & $43.24 \pm 9.12$ & $42.78 \pm 10.55$ & $50.67 \pm 6.22$ \\
\hline
\end{tabular}

Regression analyses showed that, for proxies, GDS score $(\Omega=$ $-0.269 ; \mathrm{p}=.001$ ) accounted for $8.3 \%$ of the variance of proxies' rating on Mental Summary Composite Score (MSC) $(R=0.321$, corrected $\left.\mathrm{R}^{2}=0.083\right)$, while age $(\Omega=0.019 ; \mathrm{p}=.058)$ and years of education $(\beta=-0.02 ; p=.928)$ could not be considered significant predictors. However, this model could not explain proxies' rating on Physical Summary Composite Score (PSC). Separately, this same regression model was not significant for the prediction of scores reported by participants' themselves.

Table 2 shows the results for the 12-Item Short Form Health Survey, version 2 (SF-12-v2). Both patient's and an informant's (i.e. relative or main caregiver) questionnaires were collected. In this case, the informants needed to answer to the questions as if they were the patients themselves. It is remarkable, among other measures, the low levels for Physical Role, Social Function and Emotional Role for healthy controls as reported by their proxies. As it can be seen, patients with DLB and FTD showed the lowest levels of HRQoL, while AD and HC showed the highest. These differences between groups have extensively been reported and discussed elsewhere (Onandia-Hinchado \& Diaz-Orueta, 2019).

In order to observe to what extent respondents agreed with their proxies (comparing separately cognitive decline - four dementia groups plus MCI- versus HC), Intraclass Correlation Coefficients were calculated to measure the level of respondent-proxy agreement, as shown in Table 3.

Table 3

Agreement between patients and proxies (ICC).

\begin{tabular}{lll}
\hline $\begin{array}{l}\text { HRQoL Indices and } \\
\text { Composite Scores }\end{array}$ & $\begin{array}{l}\text { Cognitive Decline Groups (MCI } \\
\text { and 4 dementia subgroups) } \\
\text { ICC }\end{array}$ & $\begin{array}{l}\text { Healthy } \\
\text { Controls } \\
\text { ICC }\end{array}$ \\
\hline Physical Function & $.877^{*}$ & $.646^{*}$ \\
Physical Role & $.703^{*}$ & $.812^{*}$ \\
Body Pain & $.810^{*}$ & $.652^{*}$ \\
General Health & $.633^{*}$ & $.605^{*}$ \\
Vitality & $.583^{*}$ & .053 \\
Social Function & $.759^{*}$ & -.309 \\
Emotional Role & $.570^{*}$ & .476 \\
Mental Health & $.544^{*}$ & .370 \\
Physical Summary & $.879^{*}$ & $.757^{*}$ \\
$\quad$ Composite Score & & .290 \\
Mental Summary Composite & $.747^{*}$ & \\
$\quad$ & & \\
\hline
\end{tabular}

${ }^{*} p=.01 ; * * p=.05$ ( $p$-value: 2 -tailed).
As it can be seen, there were many moderate to high ICC (Fleiss, 2011), especially in the groups with cognitive decline, who had a larger number of factors with agreement between patients and proxies, and of higher intensity than in healthy controls. As it can be seen in the group with cognitive decline, those agreements were higher in those components that are more tangible and visible (i.e. Physical Function, Physical Role, Body Pain, and, subsequently, in the PSC score) than in 'mental' indices. The few agreements achieved by HC group were also seen in physical indices.

Finally, knowing the agreement levels between the participants' and their proxies (separately for participants with cognitive decline and healthy controls), Table 4 compares the mean differences of discrepancy scores obtained between the groups with cognitive decline and healthy controls. As we can observe, no statistically significant differences were found between discrepancy scores of the cognitive decline groups (i.e. four dementia groups plus MCI) and HC.

\section{Discussion}

The goal of the current study was to measure self-reported Health Related Quality of Life in individuals with different types of dementia, mild cognitive impairment (MCI) and healthy controls (HC), as well as their proxies, and to assess participant-proxy agreement levels. We hypothesized, based on previous research, that discrepancies between participants and proxies across all HRQoL domains would be high and significant. However, in terms of patient-caregiver agreement, the levels of agreement are strong in the groups with cognitive decline (dementia groups plus MCI patients), which suggests that patient centred measures are reliable and are coincidental to those reported by their own caregivers, who are the ones who best know them. It is possible that early signs and symptoms of dementia in earlier stages make both patients and proxies adjust their expectations and rate decline in later stages in a more realistic way, but that assumption would require further research. This seems to contradict other authors' statements (Banerjee et al., 2006, 2009; Logsdon et al., 2002) in relation to loss of insight and its influence in HRQoL self-assessment (or, at least, it may not apply to mild to moderate stages of decline). However, in order for this reliability to be stronger, the type of variables that are more conveniently self-reported are preferably those related to physical functioning. In any case, this study supports previous studies stating the need to complement HRQoL assessment with both patients and proxies' reports (Banerjee et al., 2009).

We also observed that, attending to the percentage of variance 
Table 4

Discrepancy differences between patients with cognitive decline and their proxies, and Healthy Controls and their proxies.

\begin{tabular}{|c|c|c|c|c|}
\hline HRQoL Indices and Composite Scores & $\begin{array}{l}\text { Mean participant-proxy discrepancy. Cognitive Decline } \\
\text { Groups } \bar{X}(\mathrm{SD})\end{array}$ & $\begin{array}{l}\text { Mean participant-proxy discrepancy. Healthy Controls } \bar{X} \\
\text { (SD) }\end{array}$ & $\mathrm{t}$ & $p$ \\
\hline Physical Function & $0.19(0.79)$ & $0.15(0.99)$ & 0.222 & .824 \\
\hline Physical Role & $0.88(1.19)$ & $0.87(0.66)$ & 0.50 & .960 \\
\hline Body Pain & $0.17(1.01)$ & $-0.18(1.15)$ & 1.598 & .113 \\
\hline General Health & $0.02(1.12)$ & $-0.26(1.10)$ & 1.206 & .230 \\
\hline Vitality & $-0.27(1.41)$ & $-0.75(1.39)$ & 1.642 & .103 \\
\hline Social Function & $1.27(1.04)$ & $1.21(1.30)$ & 0.247 & .805 \\
\hline Emotional Role & $1.28(1.45)$ & $0.89(0.75)$ & 1.935 & .056 \\
\hline Mental Health & $0.40(1.21)$ & $0.09(1.07)$ & 1.241 & .217 \\
\hline Physical Summary Composite Score & $1.20(7.62)$ & $0.57(9.34)$ & 0.357 & .722 \\
\hline Mental Summary Composite Score & $9.1(9.33)$ & $5.49(9.27)$ & 1.729 & .086 \\
\hline
\end{tabular}

(p-value: 2 -tailed).

explained by cognitive decline (i.e. GDS) in caregivers' opinions, their ratings of patients' HRQoL seem to be only slightly influenced by this variable. Thus, although according to other authors' findings (Banerjee et al., 2006), age seems to have a great impact on HRQoL ratings with dementia populations (so that it could condition differences in ratings between patients and caregivers), we do not find this age related bias in our study.

Separately, the agreement between healthy controls and their proxies are weaker, with relatives reporting significantly lower rates. There are no conclusive explanations for these results, however, they may suggest a potential underdiagnosis of many pathologies affecting cognitive status and, especially, awareness or insight. In other words, it is plausible that some individuals in the HC sample (as it may happen in general population) can mask their symptomatology and try to overcompensate their deficits, which may place them under the effect of the 'disability paradox' (Albrecht \& Devlieger, 1999).

Limitations of this study are clear. Our small sample size may have not reflected the age bias shown in previous studies when it comes to rate HRQoL by proxies. In addition, reasons for discrepancies between healthy controls and proxies are only tentative, and require further analysis and research. For the proxies, the criteria of a familiarity relationship between the participant and their proxy of at least 6 months was followed (as required by the administered questionnaire), but the small sample size did not allow to differentiate between formal and informal caregivers, which would be interesting but beyond the scope of the current study. In terms of the HRQoL questionnaire used, at the time of data collection there were no normative data for the Spanish population, which explains why the American version translated to Spanish was used instead, thus leading to a more cautious interpretation of obtained data. A further consideration of the reasons for agreement in HRQoL between patients and proxies would have benefited from comparisons between patients and proxies at each dementia subgroup level, which was not possible in our study due to the small sample size in some subgroups. However, the specific differences between dementia subtypes in HRQoL ratings between patients and proxies would merit further research and could provide additional valuable information to discuss the usefulness of HRQoL rates as an additional resource to differentiate between dementia subtypes. Further studies with larger, more representative samples and with dedicated, norm-based measures for HRQoL are required, but this study opens the door to a significant further consideration of HRQoL in populations with cognitive decline, also when developing intervention programmes that focus both on cognitive and functional outcomes.

Altogether, findings of the current study represent a reaffirmation of the usefulness of HRQoL measures and the need to take them into account in clinical assessment settings, as well as in intervention contexts. Likewise, the need to take into account both patients' and proxies' perceptions about HRQoL is evident (Schwartz, 2013). In this way, it has recently been shown how the dementia course and progress is affected by variables other than those of classical cognitive nature. Thus,
Martyr et al (Martyr et al., 2018) have recently reported about the complexity of quality of life in dementia and the need to go beyond cognitive variables; more specifically, to include levels of behavioural and psychological disturbance (agitation, depression, anxiety, disinhibition, and irritability, among others) when accounting for the impact of quality of life in dementia. Findings of the current study may be of high relevance when planning for interventions that are complementary to pharmacological and cognitive rehabilitation. These envisioned interventions should target, not only the control of anxiety and depression comorbidities, but also the adaptation of patients to their disorders, adjustment of expectations, perception of functionality at all levels, and the aim to cover all basic needs. Moreover, interventions targeting disease awareness would imply a significant gain that would allow to preserve the ability to estimate one's own HRQoL for longer periods. Finally, measures of HRQoL could assist in monitoring the progress and efficacy of different treatments, supporting a higher personalization of interventions.

\section{Funding}

This study was performed with a grant from the Basque Health Innovation and Research Foundation BIOEF for the first author, reference number BIOEF10/007.

\section{Ethical approval}

This study was linked to the achievement of the $\mathrm{PhD}$ by the first author, Dr. Onandia-Hinchado, at the University of Deusto in Bilbao (Spain). The study fulfilled all the ethical requirements and obtained the required ethical approval from the University of Deusto-Bilbao (Spain).

\section{Declaration of Competing Interest}

Authors declare that there are no conflicts of interests related to this study or its publication.

\section{Acknowledgements}

Authors would like to thank Dr. Juan Mari Uterga from Basurto Hospital (Bilbao, Spain) for all his dedication to facilitate the work in the NeuroDemeNPsia Project, and, especially, in the recruitment and provision of the sample of both clinical and healthy participants.

\section{References}

Albrecht, G. L., \& Devlieger, P. J. (1999). The disability paradox: High quality of life against odds. Social Science \& Medicine, 48(8), 977-988.

Banerjee, S., Smith, S. C., Lamping, D. L., et al. (2006). Quality of life in dementia: More than just cognition. An analysis of associations with quality of life in dementia. $J$ Neurol Neurosurg Psychiat, 77(2), 146-148. 
Banerjee, S., Willis, R., Matthews, D., Contell, F., Chan, J., \& Murray, J. (2007). Improving the quality of care for mild to moderate dementia: An evaluation of the Croydon Memory Service Model. Int J Geriatr Psychiat, 22(8), 782-788.

Banerjee, S., Samsi, K., Petrie, C. D., et al. (2009). What do we know about quality of life in dementia? A review of the emerging evidence on the predictive and explanatory value of disease specific measures of health related quality of life in people with dementia. International Journal of Geriatric Psychiatry, 24(1), 15-24. https://doi.org/ 10.1002/gps.2090.

Clipp, E. P., \& George, L. K. (1993). Dementia and cancer: A comparison of spouse caregivers. Gerontologist, 33(4), 534-541.

Cole, J. C., Ito, D., Chen, Y. J., Cheng, R., Bolognese, J., \& Li-McLeod, J. (2014). Impact of Alzheimer's Disease on Caregiver Questionnaire: Internal consistency, convergent validity, and test-retest reliability of a new measure for assessing caregiver burden. Health and Quality of Life Outcomes, 12, 114. http://www.hqlo.com/content/12/1/ 114.

Dubois, B., Feldman, H. H., Jacova, C., et al. (2007). Research criteria for the diagnosis of Alzheimer's disease: Revising the NINCDS-AD RDA criteria. The Lancet Neurology, 6(8), 734-746.

Esteban y Peña, M. M., Hernández, V., Fernández, X., et al. (2010). Self- perception of health status, mental health and quality of life among adults with diabetes residing in a metropolitan area. Diabetes \& Metabolism, 36(4), 305-311.

Evans, S., \& Huxley, P. (2005). Adaptation, response shift and quality of life ratings in mentally well and unwell groups. Quality of Life Research: an International Journal of Quality of Life Aspects of Treatment, Care and Rehabilitation, 14(7), 1719-1732.

Fleiss, J. L. (2011). The design and analysis of clinical experiments. New York: Wiley.

Gwyther, L. P. (1997). The perspective of the person with Alzheimer disease: which outcomes matter in early to middle stages of dementia? Alzheimer Dis Assoc Disor, 11(Suppl. 6), 18-24 1997.

Herrman, H., Hawthorne, G., \& Thomas, R. (2002). Quality of life assessment in people living with psychosis. Social Psychiatry and Psychiatric Epidemiology, 37(11), 510-518.

Jenkinson, C., \& Layte, R. (1997). Development and testing of the UK SF-12 (short form health survey). Journal of Health Services Research \& Policy, 2, 14-18.

Karlawish, J. H., Casarett, D., Klocinski, J., \& Clark, C. M. (2001). The relationship between caregivers' global ratings of Alzheimer's disease patients' quality of life, disease severity, and the caregiving experience. Journal of the American Geriatrics Society, 49(8), 1066-1070.

Karlawish, J., Lu, M., Logsdon, R., Whitehouse, P., \& Aisen, P. (2004). How much do 12 month changes in cognition and function influence changes in mild to moderate $\mathrm{AD}$ patients and caregivers ratings of patient quality of life? Neurobiology of Aging, 25(Suppl. 2), S324.

Landeiro, F., Walsh, K., Ghinai, I., et al. (2018). Measuring quality of life of people with predementia and dementia and their caregivers: A systematic review protocol. $B M J$ Open, 8, e019082. https://doi.org/10.1136/bmjopen-2017-019082.

Logsdon, R. G., Gibbins, L. E., McCurry, S. M., \& Teri, L. (2002). Assessing quality of life in older adults with cognitive impairments. Psychosomatic Medicine, 64(3), 510-519 2002.

Lyketsos, C. G., González-Salvador, T., Chin, J. J., Baker, A., Black, B., \& Rabins, P. (2003). A follow- up study of change in quality of life among persons with dementia residing a long- term care facility. Int J Geriatr Psychiat, 18(4), 275-281.

Martyr, A., Nelis, S. M., Quinn, C., Wu, Y. T., Lamont, R. A., Henderson, C., et al. (2018). Living well with dementia: A systematic review and correlational meta-analysis of factors associated with quality of life, well-being and life satisfaction in people with dementia. Psychological Medicine, 48(13), 2130-2139.

McKeith, I. G., Dickson, D. W., Lowe, J., et al. (2005). Diagnosis and management of dementia with Lewy Bodies: Third report of the DLB Consortium. Neurology, 65(12), 1863-1872.

McKhann, G. M., Albert, M. S., Grossman, M., Miller, B., Dickson, D., \& Trojanowski, J. Q.
(2001). Clinical and pathological diagnosis of frontotemporal dementia: Report of the Work Group on Frontotemporal Dementia and pick's Disease. Archives of Neurology, 58(11), 1803-1809.

Monteagudo, O., Hernando, L., \& Palomar, J. A. (2011). Population based norms of the Spanish version of the SF-12V2 for Murcia (spain). Gaceta Sanitaria, 25(1), 50-61.

National Institute for Health and Care Excellence (2006). Donepezil. donepezil, Galantime, Rivastigmine (Review) and memantine for the treatment of alzheimer's disease. London: NICE.

Onandia-Hinchado, I., \& Diaz-Orueta, U. (2019). Health related quality of life and cognitive decline in older populations: Preliminary results from NeuroDemeNPsia study. https://doi. org/10.1177/0733464819866587.

Orrell, M., Spector, A., Thorgrimsen, S., \& Woods, B. (2005). A pilot study examining the effectiveness of maintenance Cognitive Stimulation Therapy (MCST) for people with dementia. Int J Geriatr Psychiat, 20(5), 446-451.

Rabins, P. V., \& Black, B. (2007). Measuring quality of life in dementia: Purposes, goals, challenges and progress. International Psychogeriatrics, 19(3), 401-407. https://doi. org/10.1017/S1041610207004863.

Reisberg, B., Ferris, S. H., de Leon, M. J., \& Crook, T. (1982). Global deterioration scale (GDS). The American Journal of Psychiatry, 139(9), 1136-1139.

Robles, A., Del Ser, T., Alom, J., Peña Casanova, J., \& grupo asesor del GNCD de la SEN (2002). Propuesta de criterios para el diagnóstico clínico del deterioro cognitivo ligero. La demencia y la enfermedad de Alzheimer. Neurología, 17(1), 17-32.

Sartorius, N. (1990). A WHO method for the assessment of health- related quality of life (WHOQoL). In S. Walker, \& R. Rosser (Eds.). Quality of life assessment: Key issues in the 1990s (pp. 201-207). Dordrecht: Kluwer Academic Publishers.

Schwartz, L. B. (2013). The importance of health- related quality of life in persons with cognitive impairment. Baltimore: The John's Hopkins University.

Shrout, P. E., \& Fleiss, J. L. (1979). Intraclass correlations: Uses in assessing rater reliability. Psychological Bulletin, 86(March 2), 420-428.

Sloane, P. D., Zimmerman, S., Williams, C. S., Reed, P. S., Gill, K. S., \& Preisser, J. S. (2005). Evaluating the quality of life of long- term care residents with dementia. Gerontologist, 45(Special Issue 1), 37-49.

Spector, A., Thorgrimsen, S., Woods, B., et al. (2003). Efficacy of an evidence- based cognitive stimulation therapy programme for people with dementia: Randomised controlled trial. Br J Psychiat, 183, 248-254.

Vogel, A., Mortensen, E. L., Hasselbalch, S. G., Andersen, B. B., \& Waldemar, G. (2006) Patient versus informant reported quality of life in the earliest phases of Alzheimer's Disease. Int $J$ of Geriatr Psych. 21(12), 1132-1138. https://doi.org/10.1002/gps. 1619.

Ware, J., Kosinski, M., Turner-Bowker, D. M., \& Gandek, B. (2005). How to score version 2 of the SF-12 health survey (with a supplement documenting version 1). Lincoln, RI: QualityMetric Incorporated.

Whitehouse, P. J. (2000). Harmonisation of dementia drug guidelines (United States and Europe): A report of the international working group for the harmonization of dementia drug guidelines. Alzheimer Dis Assoc Disor. 14(Supp. 1), 119-122.

Winblad, B., Brodaty, H., Gauthier, S., et al. (2001). Pharmacotherapy of Alzheimer's disease: Is there a need to redefine treatment success? International Journal of Geriatric Psychiatry, 16, 653-666.

Wlodarzcyk, J. H., Brodaty, H., \& Hawthorne, G. (2004). The relationships between quality of life, mini- mental state examination, and the instrumental activities of daily living in patients with alzheimer's disease. Archives of Gerontology and Geriatrics, 39(1), 25-33. https://doi.org/10.1016/j.achger.2003.12.004.

Woods, B., Thorgrimsen, S., Spector, A., Royan, L., \& Orrell, M. (2006). Improved quality of life and cognitive stimulation therapy in dementia. Aging \& Mental Health, 10(3), 219-226.

Zimmerman, S., Sloane, P. D., Williams, C. S., et al. (2005). Dementia care and quality of life in assisted living and nursing homes. Gerontologist, 45(Suppl. 1), 133-146. 